\title{
Reporter Genes for PET Imaging of CAR T Cells Offers Insight into Adoptive Cell Transfer
}

\author{
Benjamin M. Larimer \\ Center for Precision Imaging, Department of Radiology, Massachusetts General Hospital, Boston, Massachusetts
}

See the associated article on page 1894.

$\mathbf{I}$

mmunotherapy has drastically altered cancer treatment over the past decade. Several strategies have emerged to successfully harness the immune system as a therapeutic instrument. In lymphoma, an adoptive cell transfer approach using chimeric antigen receptor (CAR) $\mathrm{T}$ cells has received Food and Drug Administration approval for treatment in non-Hodgkin lymphoma and diffuse large B-cell lymphoma (1). In solid tumors, checkpoint blockade of PD-1 and CTLA-4 (programmed cell death protein-1 and cytotoxic $\mathrm{T}$ lymphocyte-associated antigen-4, respectively) have significantly increased durable remissions in several late-stage diseases, most notably melanoma and non-small cell lung cancer $(2,3)$. Although checkpoint blockade has demonstrated more success in solid tumors to date, a significant focus has been placed on the adaptation of CAR T cell therapy for solid tumors. Dozens of trials investigating a mixture of potential targets including epidermal growth factor receptor, human epidermal growth factor receptor 2, mucins, epithelial cell adhesion molecule, and other antigens are actively under clinical investigation. Although the promise of rationally designing a patient's own immune cells to specifically eliminate tumors is promising, CAR T therapy also can pose significant risk of severe side effects or patient death. Given the great promise and risk of such a therapy, a method to actively monitor the number of viable CAR T cells and their biodistribution is of great importance.

Although monitoring CAR T cells can be accomplished through blood tests or invasive biopsies, there are significant limitations to the amount of information that can be gleaned as compared with PET imaging methods. A blood test can assay only cells in circulation; therefore, spatial distribution of transferred cells is immeasurable and blood values do not necessarily represent tumor infiltration. In the case of a biopsy, sampling is restricted to a small part of a single site of disease, which may not be reflective of the total tumor burden. PET imaging, alternatively, offers a noninvasive, whole-body approach to detecting $\mathrm{T}$ cells. Additionally, the quantitative nature of PET allows for longitudinal studies that can inform on changes in relative levels of a CAR T cells at both the site of disease

Received Oct. 22, 2018; revision accepted Oct. 23, 2018.

For correspondence or reprints contact: Benjamin M. Larimer, Department of Radiology, Massachusetts General Hospital, Boston, MA 02114.

E-mail:blarimer@mgh.harvard.edu

Published online Nov. 15, 2018.

COPYRIGHT @ 2018 by the Society of Nuclear Medicine and Molecular Imaging. DOI: 10.2967/jnumed.118.220897 and the potential off-target sites of accumulation. There has been strong interest in imaging both $\mathrm{T}$ cells and $\mathrm{T}$ cell activation states, but these approaches target all $\mathrm{T}$ cells and would be difficult to use exclusively for CAR T cells (4-6). For adoptive cell transfer, loading of $\mathrm{T}$ cells with materials such as ${ }^{89} \mathrm{Zr}$-oxine have also been investigated; however, the labeling process has the possibility to alter the $\mathrm{T}$ cells, and as the $\mathrm{T}$ cells multiply the label is diluted, limiting the duration of longitudinal studies (7).

Although general $\mathrm{T}$ cell imaging strategies have significant value for monitoring immune-modulating therapies using the endogenous $\mathrm{T}$ cells of a patient, when exogenous and genetically engineered $\mathrm{T}$ cells are used, incorporation of a reporter gene presents an attractive alternative approach. As a demonstration of the value of CAR T reporter genes, Krebs et al. (8) establish in this issue of The Journal of Nuclear Medicine a novel and effective method for monitoring CAR T cells in vivo. The researchers incorporate a single-chain antibody designed to covalently bind to lanthanide-labeled DOTA (DAbR1) into a CD19 CAR T cell construct to create reporter gene-tagged CAR T cells. These cells were implanted in tumors or injected intravenously. DOTA was then labeled with ${ }^{86} \mathrm{Y}$ or ${ }^{177} \mathrm{Lu}$ and delivered intravenously for PET or SPECT imaging. The use of an anti-lanthanide-labeled DOTA antibody fragment provides a unique set of capabilities to CAR T cells, as PET, SPECT, or therapeutic isotopes can be delivered to the engineered immune cells. This adaptation of CAR T cell reporter genes adds to a growing number of methods for reporter genebased tracking of adoptive cell transfer. Preclinically, several approaches have used systems including the sodium iodide symporter, norepinephrine transporter, and human deoxycytidine kinase to track the tumor-homing properties and life span of T cells in several mouse models (9). Recently, Keu et al. used a reporter gene expressing herpes simplex virus type 1-thymidine kinase engineered $\mathrm{T}$ cells for longitudinal PET imaging in recurrent glioblastoma patients before and after cytotoxic lymphocyte therapy (10). Taken together, these studies demonstrate a clear path forward for clinical PET imaging of CAR T cells. It is important to develop a toolbox of strategies that use reporter genes with different levels and spatial patterns of endogenous expression and use tracers with different radioactive and blood half-lives, routes of clearance, and nonspecific accumulation.

The power of PET imaging of CAR T cells is evident in the work by Krebs et al. (8). Quantitative analysis of T cell accumulation in target tumors in addition to migration out of nontumor sites of injection are elegantly demonstrated. Although this is perhaps the most obvious benefit of reporter gene imaging of $\mathrm{T}$ cells, it is worth mentioning that only molecular imaging allows for spatially correlated surveillance of cells of interest throughout the entire body. Beyond target accumulation at a single time point, 
the residence and potential secondary sites of T cell trafficking can also be monitored by PET imaging. This could be important in understanding potential causes of therapeutic futility, in addition to the timing and location of immune-related adverse events. Finally, the use of particular reporter genes, such as DAbR1, allow for the delivery of both imaging and therapeutic isotopes, permitting selective elimination of CAR T cells in the case of a severe adverse event. Another potential use of therapeutic radioisotope labeling would be delivery of potential inflammation-inducing radiation that could recruit the patient's endogenous immune cells as the CAR T cells reach the end of their life cycle. Both cases illustrate a theranostic approach that could further enhance CAR T effectiveness.

Given both the unprecedented success of the Food and Drug Administration-approved CAR T therapies and the lack of demonstrated success of CAR T cells in solid tumors, molecular imaging offers an important insight into improving adoptive cell transfer. Reporter genes offer a genetically linked, nondisruptive way to facilitate specific targeting of PET probes to CAR T cells. This noninvasive approach enables not only single-time-point tumor quantification, but also longitudinal whole-body tracking of CAR T cells over their entire lifetime. This type of insight will be important for improving delivery, residency, and ultimately efficacy of the $\mathrm{T}$ cells. It should also help to identify and potentially treat adverse events early, before serious side effects occur. The work by Krebs et al. (8) and by others is important in identifying and developing methods that have the potential to affect immunotherapy for years to come.

\section{DISCLOSURE}

No potential conflict of interest relevant to this article was reported.

\section{REFERENCES}

1. Neelapu SS, Locke FL, Bartlett NL, et al. Axicabtagene ciloleucel CAR T-cell therapy in refractory large b-cell lymphoma. N Engl J Med. 2017;377:2531-2544.

2. Garon EB, Rizvi NA, Hui R, et al. Pembrolizumab for the treatment of nonsmall-cell lung cancer. N Engl J Med. 2015;372:2018-2028.

3. Hodi FS, O'Day SJ, McDermott DF, et al. Improved survival with Ipilimumab in patients with metastatic melanoma. N Engl J Med. 2010;363:711-723.

4. Tavaré R, Escuin-Ordinas H, Mok S, et al. An effective immuno-PET imaging method to monitor CD8-dependent responses to immunotherapy. Cancer Res. 2016;76:73-82.

5. Larimer BM, Wehrenberg-Klee E, Dubois F, et al. Granzyme B PET imaging as a predictive biomarker of immunotherapy response. Cancer Res. 2017;77:2318-2327.

6. Larimer BM, Wehrenberg-Klee E, Caraballo A, Mahmood U. Quantitative CD3 PET imaging predicts tumor growth response to anti-CTLA-4 therapy. J Nucl Med. 2016;57:1607-1611.

7. Weist MR, Starr R, Aguilar B, et al. PET of adoptively transferred chimeric antigen receptor T cells with ${ }^{89} \mathrm{Zr}$-oxine. J Nucl Med. 2018;59:1531-1537.

8. Krebs S, Ahad A, Carter LM, et al. Antibody with infinite affinity for in vivo tracking of genetically engineered lymphocytes. J Nucl Med. 2018:59:1894-1900.

9. Moroz MA, Zhang H, Lee J, et al. Comparative analysis of T cell imaging with human nuclear reporter genes. J Nucl Med. 2015;56:1055-1060.

10. Keu KV, Witney TH, Yaghoubi S, et al. Reporter gene imaging of targeted T cell immunotherapy in recurrent glioma. Sci Transl Med. 2017;9. 